\title{
The Elimination of Tinnitus with Miracurall - A Case Study
}

\author{
Abhijit Naskar \\ Inventor, Independent Research Scientist, Kolkata, India \\ email : victornskr@gmail.com
}

\begin{abstract}
Let's begin this case study with a little Jules Verne touch. To quote James Mason from the movie "Journey to The Center of The Earth" - "Madam, since the beginning of time all women have heard footsteps, up there". Well these words can be hailed partially true in modern day USA along with some other countries. Partially; because today it's not just women but men as well, are suffering from such situations of annoying noise. This medical condition is named as Tinnitus. In this paper I'm not going to discuss the etiology of Tinnitus, rather I'd attract fellow researchers towards the events of recovery in a woman's life, who has been distressed with Tinnitus for 3 years. The recovery is made possible by the very device that was invented by me to cure Diabetes Mellitus. The preliminary research paper of the device "Miracurall" depicts the pathways of resolving innumerable disorders inside the human body starting with Diabetes Mellitus. To begin explaining her recovery I must start by saying that after two days of beginning Miracurall administration, for the first time in a couple of years she had a good night's sleep without artificial sleep inducing music of rain sound cd.
\end{abstract}

Keyword:Tinnitus, Miracurall, noise, sleep, Diabetes Mellitus, USA

\section{Introduction}

Tinnitus is the perception of sound for which there is no acoustic source external to the head. It poses a significant clinical problem for millions of people in the United States and is proportionally problematic in countries where epidemiological data have been reported. In USA itself an estimated 50 million people suffer from Tinnitus. It is commonly referred to as "ringing in the ears" and often described in variety or terms like "whistling", "roaring", "chirping" etc. In some developing countries like India it is not even considered as a medical condition. Due to common ignorance of the society over there many people consider those symptoms of Tinnitus as spiritual awakening or haunting of ghosts. As this paper is not about the etiology of Tinnitus, so I'd not go deeper. In simple words we can say that it is not a disease, but a symptom that can result from a wide range of diseases. Those diseases harm the auditory system of the human body by damaging the Cranial Nerve VIII or Vestibulocochlear Nerve. Hence this damage leads to Tinnitus. Pretty obviously which means that once the damaged neural circuitry of the auditory system is healed the symptoms caused by it would also disappear. A recent case of Miracurall administration on a Tinnitus sufferer has revealed that M-Pulses (Naskar, 2014) activate the regeneration of the damaged neural network of the auditory system. Hence it reduces the Tinnitus to a great extent and eventually makes it disappear completely.

\section{Background}

\section{Case Study}

The individual whose condition is being discussed in this paper is a 63 years old female American. She has been a Tinnitus sufferer for around three years. She first noticed "pulsatile" type Tinnitus around October in the year 2011. She went through all the tests prescribed by her ENT Doctor. The tests that were prescribed for her were MRI of the head, carotid artery sonogram, physical inspections, hearing tests and others. But quite unexpectedly no eloquent reason for her condition of Tinnitus was found. This kind of situation is common in Tinnitus sufferers. No noticeable hearing loss occurred in the person. Over the years she was able to habituate with the sound which has mostly been evidenced in her left ear. Also to make the sleep a little less disrupted she has been using the sound of rain through CD player the whole night for more than a year. She has gone through many treatments throughout the years passed, but no sustainable impact over her Tinnitus has been noticed.

\section{Results of Miracurall Administration}

She started administering Miracurall on $4^{\text {th }}$ August, 2014 according to specific protocol meant for the condition of Tinnitus. After the very first administration of the Miracurall she noticed a remarkable reductionin the sound of Tinnitus. Since her Auditory System has been used to the sound of Tinnitus for a long time, the fading of its intensity lead to a perception of the faintness of the Tinnitus memory, more like a phantom sound. Since the day she started administering Miracurall the sound of Tinnitus has been diminishing with less frequent occurrence concentrated in the left ear.As explained on $7^{\text {th }}$ Augustby herself, the intensity of the Tinnitus sound 
increases after 3 am but before 5 am it starts fading. For the first time in a couple of years she did not need to turn on the CD player throughout the night, there was only the sound of the fan. There were several other symptoms that she felt in the early days of administration, like pressure in the head, neck and arms. At times after the session of Miracurall her diastolic blood pressure reading increases a bit too. The etiology of these symptoms are quite interesting. The recovery of the neuronal network requires an increase in blood circulation. So, during the administration she sometimes feels pressure in the head, neck and arms, which is the pathway of M-Pulses passing to the brain. When the damage in the neuronal network starts to heal, naturally increased blood circulation leads to a bit elevated blood pressure. Her protocol of Miracurall administration was to use three consecutive days followed by one day rest, but due to her symptoms of increased blood pressure at times, her protocol was changed to one day of usage followed by a day of rest. The change of protocol has increased the rate of recovery. As explained on $16^{\text {th }}$ August by herself, Miracurall helped lessen the sound to such extent that she only hears a faint whisper sometimes. Miracurallhas had a noticeable and sustainable effect of diminishing the phantom sounds along with frequentelimination of her Tinnitus throughout the day. Based on her rate of recovery, as she continues the administration the damage in her Auditory System will get completely healed and her Tinnitus would be eliminated for good.

\section{Summary}

Since Tinnitus is not really a disease it is much easier to curethan any other typical disorders like Miracurall's fist adversary Diabetes Mellitus. Tinnitus is just a symptom resulting from a damaged Auditory System. Hence, once the damaged neural circuitry is healed, the symptoms connected to it disappear naturally. The case study of this paper reveals the positive effect of Miracurall on curing the phantom sound of Tinnitus, which indicates that further research on this path would lead to the healthy impact of Miracurall on Phantom Limb Pain as well. Tinnitus is the second step of Miracurall after Diabetes Mellitus, towards the discovery of its wide range of curative properties.

\section{Acknowledgment}

I thank Bonnie Bernard for her whole hearted participation in this experiment. Miracurall's healthy impact on her symptoms of Tinnitus so far, would shed a ray of hope upon 50 million Americans and millions of others suffering from Tinnitus around the world.

\section{References}

[1]. Abhijit Naskar (2014), Miracurall The Electronic Cure For Diabetes Mellitus

[2]. V.S. Ramachandran and Sandra Blakeslee (1998), Phantoms in the Brain

[3]. Richard S. Snell (2010), Clinical Neuroanatomy 